\title{
Componentes del capital humano: Indicadores y perspectivas de medición
}

\section{Human Capital components: measuring indicators and outlooks}

\author{
Lic. Mahali Adlai Zazueta Trejo \\ Instituto Tecnológico de Sonora, México \\ Mahali.zazueta@gmail.com
}

\author{
Dr. Carlos Armando Jacobo Hernández \\ Instituto Tecnológico de Sonora, México \\ cjacobo@itson.edu.mx
}

\author{
Dr. Sergio Ochoa Jiménez \\ Instituto Tecnológico de Sonora, México \\ sochoa@itson.edu.mx
}

\section{RESUMEN:}

Se llevó a cabo el presente estudio con el objetivo de describir los componentes e indicadores del capital humano, esto con la finalidad de tener una concepción más clara de cómo se identifica dicha variable para su desarrollo y medición. realizándose mediante el método de revisión de literatura, obteniendo como principales indicadores que se han estudiado a nivel empresarial/organizacional componentes para su medición tales como la educación, salud, experiencia, capacitación, capacidades, habilidades, conocimientos, actitudes y saberes en general de los individuos, y en cuanto a los indicadores a nivel país o región, entre los componentes más representativos se encuentran los datos demográficos de la población, inversión en la educación y salud, instituciones de educación y salud, producto interno bruto, tasa de salarios y oportunidades laborales.

\section{ABSTRACT:}

This research was carried out to describe the components and indicators of human capital with the aim of having a clearer vision of the way to identify the said variable for its development and measurement. To this effect, the method of literature review was used and measuring components such as education, health, experience, training, skills, abilities, knowledge, attitudes and general know-how of individuals were obtained as main indicators that have been studied at the business and organizational level. As for indicators at country or regional level, among the most representative components are the demographic data, education and health investment, educational and health institutions, gross domestic product, wage rate and labor opportunities.

\section{RESUMO:}

O presente estudo foi realizado com o objetivo de descrever os componentes e indicadores do capital humano, a fim de ter uma concepção mais clara de como essa variável é identificada para seu desenvolvimento e medição. Foi realizado com o método de revisão de literatura e se obteve, como principais indicadores estudados no nível empresarial / organizacional, componentes para sua medição como educação, saúde, experiência, treinamento, capacidades, habilidades, conhecimentos, atitudes e saberes em geral dos indivíduos. Em relação aos indicadores em nível de país ou região, entre os componentes mais representativos encontram-se os dados demográficos, investimento em educação e saúde, instituições de educação e saúde, produto interno bruto, taxas de salários.

\section{RÉSUMÉ:}

Cette étude a été réalisée pour décrire les composantes et les indicateurs du capital humain, ceci dans le but d'avoir une vision plus claire de la manière d'identifier cette variable pour son développement et sa mesure. La méthode de révision de documentation a été utilisée et des composantes de mesure, telles qu'éducation, santé, expérience, formation, capacités, compétences, connaissances, attitudes et savoirs des individus en général ont été obtenues comme indicateurs principaux ayant été étudiés au niveau commercial et organisationnel. Quant aux indicateurs au niveau pays ou région, parmi les composantes les plus représentatives, il y a les données démographiques, l'investissement en éducation et santé, les institutions éducatives et de santé, le produit intérieur brut, le taux de salaire et les opportunités de travail.

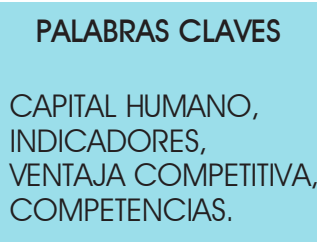

\section{PALABRAS CLAVES}

CAPITAL HUMANO,

NDICADORES

COMPETENCIAS

\section{KEYWORDS}

HUMAN CAPITAL, INDICATORS, COMPETITIVE EDGE, COMPETENCIES

\section{PALAVRAS CHAVES \\ CAPITAL HUMANO, INDICADORES, \\ VANTAGEM \\ COMPETITIVA, COMPETÊNCIAS}

\section{MOTS CLÉS}

CAPITAL HUMAIN, INDICATEURS, AVANTAGE CONCURRENTIEL, COMPETENCES 


\section{INTRODUCCIÓN}

En la actualidad el valor que posee cualquier entidad u organización ya no puede ser solo determinada por sus activos tangibles, es decir, recursos materiales, económicos, de infraestructura, entre otros, sino también por la generación y desarrollo de conocimientos, habilidades y capacidades de tales agentes denominados como capital humano $(\mathrm{CH})$ el cual puede considerarse un valor intangible que puede llegar a ser clave para obtener ventajas competitivas en cualquier tipo de organización o incluso hasta nivel país (Lizote, Ribeiro, Verdinelli y Terres, 2017).

De acuerdo con Greer, Lusch y Hitt (2017) es posible implementar diversas estrategias en las organizaciones mediante el desarrollo y potenciación del $\mathrm{CH}$, y debido a esto es tan importante, incluso para la implementación de alguna estrategia dentro de una organización, que los directivos o alta gerencia sean capaces de involucrar a los individuos que componen toda la entidad organizativa.

De igual manera, Cardona, Montes, Vásquez, Villegas y Brito (2007) mencionan que desde la aparición del CH como tal en la década de 1960, ha evolucionado y tomado diferentes definiciones según los autores que lo han trabajado, variando así sus componentes y alcances, destacando como los componentes típicos del CH: la educación formal, capacitación, la salud y las experiencias de una determinada persona que la hacen productiva y competente dentro de la organización a la cual pertenece.

Ahora bien, López, Ojeda y Ríos (2017) plantean que el capital humano, es lo más valioso para cualquier organización, y siendo de esta manera es que las empresas deben de proponer las medidas necesarias para propiciar y favorecer al desarrollo de este, tanto de manera profesional como personal, pues además el CH es la base para su crecimiento empresarial y clave importante para el posicionamiento de la organización en el mercado.

Así mismo, Münch (2017) menciona que el capital humano es de gran importancia debido a que implica conseguir diversos beneficios para las organizaciones, tales como el aumento de la eficiencia, eficacia, calidad, optimización de los recursos tecnológicos, intelectuales, materiales y financieros, promoviendo además un clima propicio y mejora en la calidad de vida de los individuos que conforman la organización, así como sus niveles de motivación.

Analizando lo anterior, se logró identificar en un primer nivel de análisis de revisión de literatura, que existían comúnmente dos vertientes estudiadas en torno a los indicadores de $\mathrm{CH}$, tales como a nivel organización/empresa y País/región, por lo que en este punto es importante hacer mención del hecho de que se pueden encontrar estas diferentes formas de medirlo, y por ende reflexionar acerca de la confusión que podría crear esta situación en relación a los indicadores que existen para determinar el nivel de desarrollo de $\mathrm{CH}$.

Por lo que considerando lo anterior, para la elaboración del presente artículo se plantea como objetivo describir los componentes e indicadores del capital humano, mediante una profunda revisión de literatura de artículos en los que se han propuesto tales indicadores, con la finalidad de tener una concepción más clara de cómo se identifica dicha variable para su desarrollo y medición.

Tal interés es debido a que se ha encontrado que el $\mathrm{CH}$ funge en la actualidad como un elemento clave para el desarrollo de nuevas capacidades, conocimientos, habilidades, e incluso posicionamiento, productividad y aumento económico en todo tipo de organización y a su vez impactando a nivel región o hasta país, tal como menciona Raffiee y Coff (2016) tomando como base la teoría del capital humano, en donde argumenta que según expertos en estrategia han determinado al capital humano específico de la empresa como un fuerte factor de ventaja competitiva.

Además, es por esta razón que resulta interesante describir como se ha abordado el término y los componentes del capital humano, por lo que en este punto resulta relevante mencionar a algunos autores clave que han acuñado dicho concepto sobre el tema estudiado, tales como Theodore William Schultz (1902-1998), Jacob Mincer (19222006) y Gary S. Becker (1930-2014), esto con la intención de contribuir al logro del objetivo propuesto, identificando indicadores y/o componentes del CH desde sus inicios a la actualidad. 


\section{DESARROLLO}

De acuerdo con Pérez y Castillo (2016) fue Adam Smith (1723-1790) el primero en exponer el término de Capital humano, siendo a partir de ese momento retomado por otros autores como Schultz y Becker, estableciéndose formalmente la teoría económica de capital humano o simplemente teoría del capital humano, a la cual se le atribuyen comúnmente variables en relación a las capacidades y experiencia del individuo. Por otro lado, Sweetland (1996) argumenta que la teoría del capital humano plantea que tanto la sociedad e individuos en general obtienen un beneficio de índole económico, este proveniente de las inversiones y desarrollo de las personas.

\section{Conceptualización del capital humano}

Considerando lo anteriormente mencionado, Schultz (1961, citado en Cardona, et al., 2007) argumenta que cuando se invierte en educación, salud, crianza, formación de profesionales, investigación, etcétera, se está invirtiendo realmente en recurso humano, lo cual a futuro traerá diversos beneficios e impacto no solo a nivel organización, sino también a nivel región o país, pues entre los beneficios se encuentra la disminución de la brecha entre pobres y ricos, trayendo consigo una calidad de vida equitativa.

De igual manera, Becker (1983, citado en Aronson, 2007) define al capital humano al igual que Schultz como una inversión, la cual realizan las organizaciones en desarrollar la creación de conocimientos y formación de las personas tanto personal como profesional, lo cual a su vez permitirá generar un mayor rendimiento y productividad en la economía moderna. Así mismo, Mincer (1974, citado en Herrera, 2010) menciona que el nivel de desarrollo de Capital Humano de cada individuo, determina que tan productivo será este en el puesto que desempeñe dentro de la organización, además menciona que entre mayor nivel de educación o formación posea el individuo deberá de obtener mayor retribución económica.

De acuerdo con Potelienè y Tamašauskienè (2014) el capital humano vendría a ser compuesto por elementos tales como el conocimiento personal, las habilidades, la educación, las habilidades innatas, la experiencia, las actitudes, el comportamiento, la inteligencia, la creatividad, el espíritu empresarial, la motivación, la innovación, la perspicacia, la experiencia, el estado físico, emocional, mental y de la salud entre los más representativos, lo cual contribuye a aumentar la productividad y por ende los ingresos de un individuo.

Incluso Ganga, Cassinelli, Díaz y Maluk (2016), mencionan que el término de capital humano, puede ser visto como el valor económico potencial que se puede derivar de la capacidad productiva de un individuo, debido a que dicha capacidad tiene un impacto en la eficiencia y rentabilidad de las organizaciones, que a su vez las lleva a obtener mejores resultados que después se definen en mayores ingresos.

Así mismo Rocha, Genari y Macke (2018) argumentan que "el capital humano contempla el conjunto de habilidades, capacidades y conocimientos de cada trabajador, caracterizándose como un importante elemento para fomentar la capacidad de innovación y la creación de ventajas competitivas sostenibles" (p. 514).

\section{El capital humano en las organizaciones}

Los autores García y Chávez (2017) realizaron una investigación con el objetivo de analizar indicadores que se deben de tener en cuenta para medir el CH en micro, pequeñas y medianas empresas (MIPYMES), ya que dicha variable ha tomado importancia como medio para que las organizaciones logren obtener mayor eficiencia, productividad y sobre todo crecimiento, argumentando además que estos contribuyen a evaluar el nivel de desarrollo del $\mathrm{CH}$ a nivel local o regional desde una perspectiva de medición mayor.

En una investigación llevada a cabo por Chadwick (2017) se planteó como objetivo el describir una serie de elementos potenciales del $\mathrm{CH}$, con la finalidad de que las empresas los tomaran en cuenta para su desarrollo y aprovechamiento, 
como principal sustento del estudio, se argumentó que para que una empresa obtenga rentas de capital humano, esta deberá aprovechar las capacidades y recursos al máximo, lo cual hace referencia a que el CH para una organización en sí es una renta, debido a que no es algo que la empresa pueda comprar o poseer, ya que los individuos que conforman podrían dejarla en cualquier momento, llevándose con ellos el conocimiento generado.

En el contex to educativo, Nnebedum y Akinfolarin (2017) llevaron a cabo un estudio argumentando que debido al bajo desempeño de exámenes en estudiantes de secundaria en el estado de Ohio estados unidos, era necesario investigar las prácticas para el desarrollo del capital humano de directores, con el fin de que estos mejoraran la administración del personal docente y con ello la entrega educativa, de acuerdo a los hallazgos encontrados, se argumenta la necesidad de que el gobierno otorgue subsidios anuales a los directores para el patrocinio de talleres, seminarios y conferencias, con el fin de mantenerse actualizados y adquirir nuevos conocimientos que los ayuden adaptarse a las nuevas prácticas educativas.

Por otra parte, Israelsen y Yonker (2017) mencionan que las empresas que solo potencian una pequeña parte de su capital humano denominado como "clave", es decir solo algunas personas en determinados puestos, se exponen a perder dicho conocimiento generado específicamente en ese personal que le agrega cierto valor y ventaja a la empresa, pues la salida de alguno de estos empleados es más costosa que perder numerosos empleados eventuales, haciendo difícil el remplazarlos. Debido a lo anterior, entonces lo más conveniente sería que la organización establezca siempre como aplicable cada conocimiento generado a toda la organización, evitando así la pérdida de tales elementos intangibles tan valiosos y potenciados por ella misma.

Siguiendo con una investigación en relación a lo anterior, se llevó a cabo un estudio en centros de investigación, con la intención de proponer medidas que eviten la fuga del capital humano, debido a que se ha encontrado que a este no se le permite desarrollarse plenamente, dándosele más importancia al trabajo en sí, sin propiciar la motivación, la creatividad, la iniciativa, etcétera. Como principal hallazgo se pudieron determinar cuatro indicadores principales del $\mathrm{CH}$, como lo son: conocimientos, experiencias, creatividad e iniciativa (Bermúdez, Boscan, Muñoz, Vidal y Archila, 2017).

\section{El capital humano aplicado a regiones y países para su desarrollo.}

En una investigación realizada por Giménez, López y Sanaú (2014) propusieron como objetivo identificar indicadores del capital humano para su medición desde dimensiones cualitativas y cuantitativas, para a su vez determinar la relación con el crecimiento del PIB y la innovación a nivel país, para lograr el objetivo planteado se aplicó el test de Granger a una muestra conformada por 15 países de la Organización para la Cooperación y el Desarrollo Económicos (OCDE), en donde los principales hallazgos encontrados determinan que el desarrollo de indicadores precisos, que cuantifiquen las habilidades y las diferencias de productividad que existen en el mercado laboral, deberían constituir un objetivo clave de la política económica.

Debido a la importancia de las externalidades del capital humano para los diferenciales salariales en regiones de Alemania, Heuermann (2011) realizó un estudio en donde se muestra que dichos efectos salariales se relacionan con el CH del país y se debe a las mejores oportunidades laborales, el sistema de aprendizaje alemán, al nivel de conocimientos, habilidades generales y al nivel general alto de educación existente.

Por otro lado en una investigación realizada por Munirovich, Ilfarovich, Olegovych, Sergeevna, Sultangalievich, Andreevich y Mikhailovna (2018) se buscó determinar el valor del capital humano, su potencial, así como sus principales componentes, con el fin de proponer una escala de evaluación del CH, la cual contribuya a la formación y desarrollo de programas destinados a mejorar el bienestar social y económico de Rusia y sus regiones, ya que es considerado un factor clave para el desarrollo de la economía, que no solo promueve el aumento de la productividad laboral, sino también a la generación de nuevos conceptos e innovaciones. 
Así mismo, en un estudio realizado a 166 agricultores pequeños en todas las regiones de Sudáfrica, Thindisa y Urban (2018) buscaron determinar en qué grado la participación en el agro procesamiento es determinada por factores del capital humano y social. Entre los principales hallazgos encontrados de dichos factores, se muestra una influencia positiva y significativa en la participación del proceso agrícola, por lo que finalmente recomendaron que los responsables de formular políticas para dichos procesos deben de ser conscientes de cómo los factores del $\mathrm{CH}$ tales como la educación y la experiencia previa, impactarán en las actividades realizadas, lo cual a su vez ayudara a disminuir los costos de transacción.

En otra investigación, se analizó el efecto de la inversión en capital humano en países asiáticos, en relación a su crecimiento económico, desigualdad y equilibrio fiscal, ya que según estudios anteriormente revisados sugieren que el $\mathrm{CH}$ influye positivamente en la productividad laboral y por ende en el producto, dichos estudios también mostraron que tal efecto positivo es más fuerte para los más pobres y por lo tanto es beneficioso para la equidad de nivel socioeconómico en los países asiáticos (Abrigo, Lee, y Park, 2017).

Debido a la creciente integración económica, la globalización y la apertura internacional, es que va en aumento la competitividad de los países y regiones, como un ejemplo de lo anterior Maciulyte y Matuzeviciute (2018) realizaron una investigaron sobre el impacto del capital humano en la productividad del trabajo de estados pertenecientes a la unión Europea, encontrando que gracias al desarrollo del capital humano es que la productividad aumenta, principalmente gracias a los indicadores de educación y salud.

De la misma forma, Villarreal (2016) llevó a cabo un estudio con el propósito de exponer las externalidades del capital humano en México, basándose primeramente en la literatura sobre el tema y datos duros, esto debido a que según estas externalidades son factores clave para el crecimiento económico de las ciudades, regiones y países, argumentando además que la teoría del capital humano demuestra que entre mayor sea el nivel educativo de la población, esto traerá consigo efectos positivos en el ámbito económico, social y político.

\section{METODOLOGÍA}

Para el desarrollo de la presente investigación se utilizó el método de revisión de literatura de investigaciones, que de acuerdo con Fink (2014) dicho método contribuye a la evaluación y síntesis de trabajos de investigación, y plantea además que al analizar estos estudios pasados sobre un tema de interés, como lo es en este caso los componentes del capital humano, es posible aportar información y datos relevantes que puedan suponer una base para futuras investigaciones. Por lo que para llevar a cabo este estudio se optó por realizar una revisión profunda de artículos de investigación científica de las diferentes bases de datos a las que se tuvo acceso, tales como Redalyc, ScieELO, Dialnet, EBSCO, Emerald, Elsevier, JSTOR y Wiley, analizando y recopilando la información más relevante para el desarrollo de este trabajo, con el fin de proponer como principal aporte los componentes e indicadores del CH más representativos.

\section{RESULTADOS Y DISCUSIÓN}

Como se planteó en un inicio, se lograron identificar dos vertientes estudiadas en torno a los indicadores de CH. La primera de ellas aborda los indicadores a nivel organización o empresa, mientras que la segunda vertiente se relaciona con indicadores a nivel País o región. A continuación se presentan los principales indicadores encontrados al interior de las organizaciones (ver tabla 1) y regiones o países (ver tabla 2), dichas tablas contienen el autor, componente, variable y finalmente los rubros medibles para el desarrollo del $\mathrm{CH}$. 


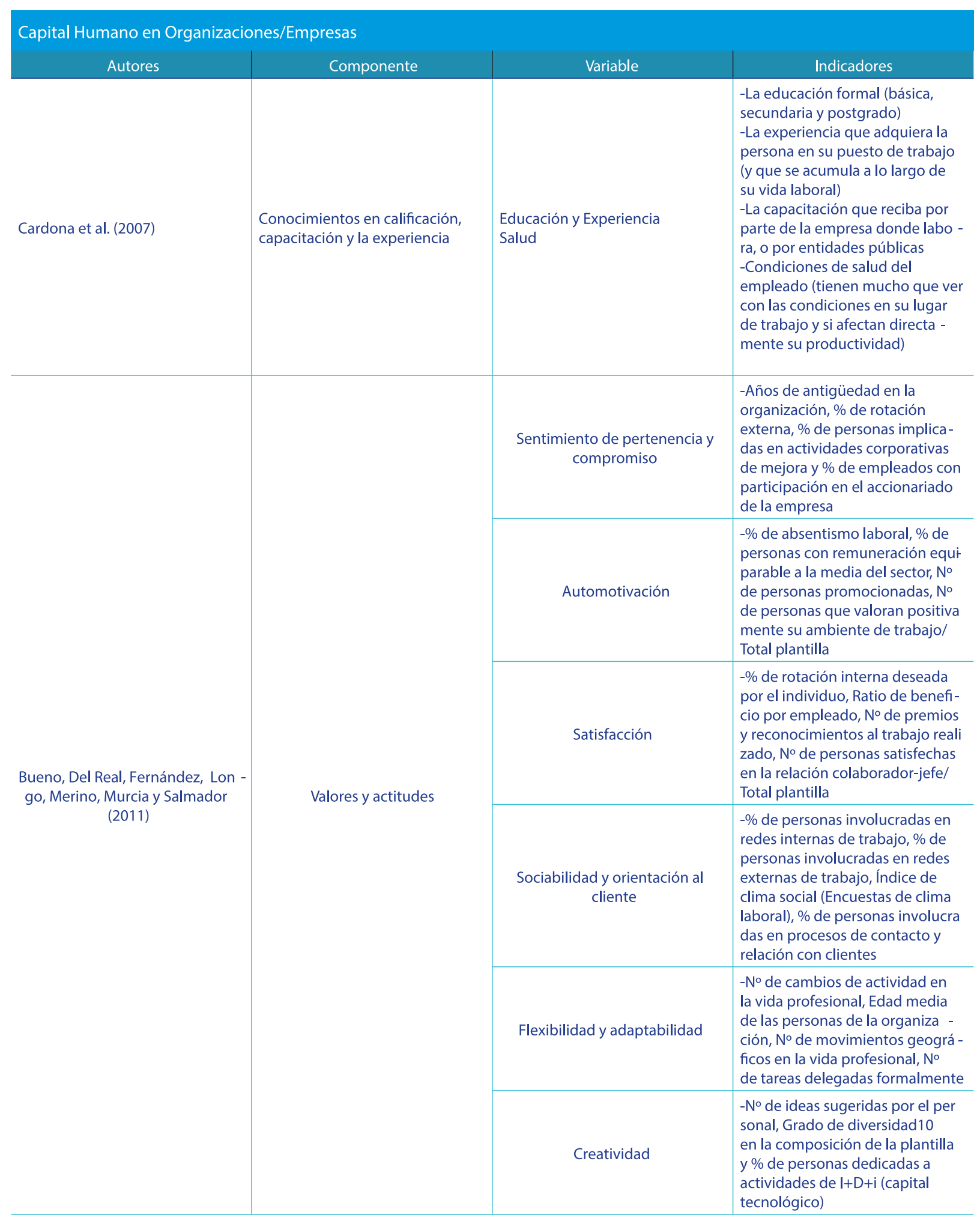




\begin{tabular}{|c|c|c|}
\hline \multirow{5}{*}{ Aptitudes } & Educación reglada & $\begin{array}{l}\text {-\% de personal con titulación } \\
\text { superior, \% de personas con otras } \\
\text { titulaciones oficiales, Media de } \\
\text { antigüedad de la titulación y } \\
\text { Grado de heterogeneidad de la } \\
\text { formación académica (Ejemplo: } \\
N^{\circ} \text { de titulados en humanidades } \\
\text { / Total de titulados; No de inge - } \\
\text { nieros / Total de titulados, etc.) }\end{array}$ \\
\hline & Formación & $\begin{array}{l}\text {-\% de personal con estudios de } \\
\text { especialización y posgrado }\end{array}$ \\
\hline & Formación interna & $\begin{array}{l}\text { - } \mathrm{N}^{\circ} \text { de personas formadas } \\
\text { anualmente / Total plantilla, No } \\
\text { de personas que han realizado } \\
\text { cursos de formación continua / } \\
\text { Total plantilla, } \mathrm{N}^{\circ} \text { de acciones } \\
\text { en el campus corporativo virtual } \\
\text { y } \mathrm{N}^{\circ} \text { de acciones formativas en } \\
\text { la intranet / } \mathrm{N}^{\circ} \text { total de acciones } \\
\text { formativas }\end{array}$ \\
\hline & Experiencia & $\begin{array}{l}\text {-Antigüedad media de experien- } \\
\text { cia en el puesto de trabajo, Anti - } \\
\text { güedad media de experiencia en } \\
\text { el sector, Antigüedad media de } \\
\text { experiencia en la organización y } \\
N^{\circ} \text { de personas con experiencia } \\
\text { profesional internacional }\end{array}$ \\
\hline & Desarrollo personal & $\begin{array}{l}\text {-\% de personas con actividades } \\
\text { socio-culturales al margen de su } \\
\text { vida profesional, \% de personas } \\
\text { con responsabilidades familiares } \\
\text { y No medio anual de promocio - } \\
\text { nes en la plantilla }\end{array}$ \\
\hline \multirow{3}{*}{ Capacidades } & Aprendizaje & $\begin{array}{l}\text {-Tiempo dedicado a actividades } \\
\text { de formación/ Tiempo total } \\
\text { de trabajo \% de trabajadores } \\
\text { que reciben formación y \% de } \\
\text { cumplimiento de los objetivos de } \\
\text { la formación }\end{array}$ \\
\hline & Colaboración (trabajo en equipo) & $\begin{array}{l}\text { - No de personas que participan } \\
\text { en dos o más equipos internos de } \\
\text { trabajo, No de personas que par - } \\
\text { ticipan en dos o más equipos ex- } \\
\text { ternos de trabajo, } N^{\circ} \text { de equipos } \\
\text { transversales y multifuncionales } \\
\text { en marcha, No de objetivos indi - } \\
\text { viduales integrados en objetivos } \\
\text { generales de equipo y Equipos } \\
\text { con incentivos compartidos en } \\
\text { relación al total de equipos }\end{array}$ \\
\hline & $\begin{array}{l}\text { Comunicación (intercambio de } \\
\text { conocimiento) }\end{array}$ & $\begin{array}{l}\text { - No de aportaciones sugeridas, } \\
\mathrm{N}^{\circ} \text { de personas involucradas en } \\
\text { plataforma tecnológica/ Total } \\
\text { plantilla, } \mathrm{N}^{\circ} \text { de aportaciones } \\
\text { llevadas a la práctica/ Total } \\
\text { aportaciones }\end{array}$ \\
\hline
\end{tabular}




\begin{tabular}{|c|c|c|c|}
\hline Autores & Componente & Variable & Indicadores \\
\hline & & $\begin{array}{l}\text { Conciliación de la vida laboral y } \\
\text { familiar. }\end{array}$ & $\begin{array}{l}\text {-\% de personas con responsabi- } \\
\text { lidades familiares, } \% \text { de horas } \\
\text { laborales ejecutadas fuera del } \\
\text { lugar habitual de trabajo, } N^{\circ} \text { de } \\
\text { personas que participa en un pro- } \\
\text { grama de conciliación y } N^{\circ} \text { de } \\
\text { medidas y servicios para facilitar } \\
\text { la conciliación }\end{array}$ \\
\hline & & Liderazgo & $\begin{array}{l}\text {-No de personas satisfechas } \\
\text { con sus responsables directos, } \\
\% \text { de personas que conocen la } \\
\text { estrategia de la organización, } \\
\% \text { de personas satisfechas en su } \\
\text { puesto de trabajo, Porcentaje de } \\
\text { personas implicadas en activida - } \\
\text { des corporativas de mejora }\end{array}$ \\
\hline \multirow{12}{*}{ Iwamoto y Takahashi (2015). } & \multirow{3}{*}{ Competencias } & Estructura de los empleados & $\begin{array}{l}\text {-Edad promedio, Duración pro- } \\
\text { medio del servicio, Porcentaje de } \\
\text { trabajador temporal, Porcentaje } \\
\text { de empleados menores de } 30 \\
\text { años en } 30,40,50,60,60, \\
\text { Porcentaje de empleados }\end{array}$ \\
\hline & & Sistemas internos & $\begin{array}{l}\text {-Esquema de incentivos para } \\
\text { la adquisición de certificación } \\
\text { técnica y técnica, programa } \\
\text { de estudios, programa en el } \\
\text { extranjero, programa de apoyo a } \\
\text { la carrera }\end{array}$ \\
\hline & & Empleo & $\begin{array}{l}\text {-Nuevo porcentaje de gradua - } \\
\text { dos, porcentaje de media carrera }\end{array}$ \\
\hline & \multirow{7}{*}{ Actitud } & Estructura de los directivos & $\begin{array}{l}\text {-Índice de gestora femenina, ratio } \\
\text { de ejecutiva femenina }\end{array}$ \\
\hline & & Estructura de los directores & $\begin{array}{l}\text {-Directores que sirven simultánea } \\
\text { mente como porcentaje de fur } \\
\text { cionarios, El número de directores } \\
\text { externos }\end{array}$ \\
\hline & & Sistemas internos & $\begin{array}{l}\text {-Medio día de vacaciones pa - } \\
\text { gas, sistema de emprendedores, } \\
\text { sistema interno de reclutamiento } \\
\text { de personal, agencia libre, } \\
\text { plan de recompensa por logros } \\
\text { especiales, opción de compra de } \\
\text { acciones }\end{array}$ \\
\hline & & Horas extraordinarias y pagadas & $\begin{array}{l}\text {-Promedio mensual de horas } \\
\text { extraordinarias, pago por horas } \\
\text { extraordinarias, días promedio de } \\
\text { vacaciones pagadas adquiridas, } \\
\text { tasa de adquisición de vacacio - } \\
\text { nes pagadas }\end{array}$ \\
\hline & & Influencia & -Relación accionaria del director. \\
\hline & & Diferencia & -Diferencial salarial \\
\hline & & Volumen de negocios & $\begin{array}{l}\text {-Tasa de rotación de empleados } \\
\text { en sus primeros tres años, tasa de } \\
\text { rotación de empleados }\end{array}$ \\
\hline & \multirow{2}{*}{ Competencia y actitud } & Pago & $\begin{array}{l}\text {-Compensación del Director, } \\
\text { Pago }\end{array}$ \\
\hline & & Pago y bienestar & $\begin{array}{l}\text {-Gastos de personal * por } \\
\text { empleado. }\end{array}$ \\
\hline
\end{tabular}




\begin{tabular}{|c|c|c|c|}
\hline Autores & Componente & Variable & Indicadores \\
\hline & Otros & El número de personas & $\begin{array}{l}\text {-El número de empleados, El } \\
\text { número de directores (escala } \\
\text { ajustada) }\end{array}$ \\
\hline \multirow{5}{*}{ Bermúdez et al. (2017) } & \multirow{5}{*}{ Indicadores del $\mathrm{CH}$} & conocimientos & $\begin{array}{l}\text {-saber cómo, educación, califi - } \\
\text { cación vocacional, conocimiento } \\
\text { relacionado con el trabajo }\end{array}$ \\
\hline & & experiencias & $\begin{array}{l}\text {-Valoración ocupacional, valora - } \\
\text { ción sicométrica, competencias } \\
\text { relacionadas con el trabajo, }\end{array}$ \\
\hline & & Creatividad & $\begin{array}{l}\text {-espíritu emprendedor, capacidad } \\
\text { de innovar, }\end{array}$ \\
\hline & & Iniciativa & $\begin{array}{l}\text {-Habilidades proactivas y reacti - } \\
\text { vas, capacidad de cambiar, con } \\
\text { los resultados }\end{array}$ \\
\hline & & Generales & $\begin{array}{l}\text {-Número de empleados, edad } \\
\text { media, gastos de personal }\end{array}$ \\
\hline \multirow{5}{*}{$\begin{array}{l}\text { Demuner, Saavedra y Camarena } \\
\text { (2017). }\end{array}$} & \multirow{5}{*}{ Indicadores descriptivos } & Experiencia & $\begin{array}{l}\text {-Antigüedad media (años), anti } \\
\text { güedad media (años) / } 40 \text { años } \\
\text { (vida profesional) (\%) }\end{array}$ \\
\hline & & Diversidad & $\begin{array}{l}\text {-Desglose por sexo: Hombres } \% \\
\text { y mujeres } \% \text {, personas con titula } \\
\text { ción universitaria } \%\end{array}$ \\
\hline & & Capacidad y desarrollo & $\begin{array}{l}\text {-Personas que han recibido forma } \\
\text { ción (\%), número medio de horas } \\
\text { de formación por persona forma- } \\
\text { da, inversión en formación sobre } \\
\text { la masa salarial (\%), inversión } \\
\text { total en formación, inversión en } \\
\text { formación por persona formada } \\
\text { (euros) }\end{array}$ \\
\hline & & Compromiso y motivación & $\begin{array}{l}\text {-Índice de rotación externa } \\
\text { (calculado con plantilla media), } \\
\text { personas con retribución flexible } \\
\text { (\%), incorporaciones en el último } \\
\text { año/plantilla total (\%) }\end{array}$ \\
\hline & & Productividad & $\begin{array}{l}\text {-Aportación al PIB por empleado, } \\
\text { recursos de clientes por em- } \\
\text { pleado, inversión crediticia por } \\
\text { empleado }\end{array}$ \\
\hline \multirow{4}{*}{$\begin{array}{c}\text { Mubarik, Chandran y Devadason } \\
\text { (2017). }\end{array}$} & \multirow{4}{*}{$\begin{array}{l}\text { Medición del capital humano en la } \\
\text { pequeña y mediana manufactura }\end{array}$} & Educación & $\begin{array}{l}\text {-Nivel de educación, Calidad de } \\
\text { la educación, Educación técnica. }\end{array}$ \\
\hline & & Experiencia: & $\begin{array}{l}\text {-Experiencia similar en la } \\
\text { industria, Experiencia laboral, } \\
\text { Tenencia organizacional }\end{array}$ \\
\hline & & Capacitación: & $\begin{array}{l}\text {-Capacitación en el trabajo, } \\
\text { Gastos en capacitación, Tiempo } \\
\text { en la capacitación, Capacitación } \\
\text { técnica, Capacitación en habi - } \\
\text { lidades blandas, Capacitación } \\
\text { previa }\end{array}$ \\
\hline & & Habilidades personales: & $\begin{array}{l}\text {-Creatividad, Inteligencia, Diversi- } \\
\text { dad, Liderazgo, Toma de riesgos. }\end{array}$ \\
\hline
\end{tabular}




\begin{tabular}{|c|c|c|c|}
\hline Autores & Componente & Variable & Indicadores \\
\hline & & Habilidades: & $\begin{array}{l}\text {-Habilidades relacionadas con el } \\
\text { trabajo, Habilidades de resolu - } \\
\text { ción de problemas, Habilidades } \\
\text { de comunicación, Habilidades } \\
\text { técnicas, Habilidades intraempre- } \\
\text { sariales }\end{array}$ \\
\hline & & Actitud: & $\begin{array}{l}\text {-Cooperación, Motivación, Com - } \\
\text { promiso, Satisfacción }\end{array}$ \\
\hline & & Estabilidad: & $\begin{array}{l}\text {-Absentismo, Longevidad, Rota } \\
\text { ción de personal }\end{array}$ \\
\hline & & Salud: & $\begin{array}{l}\text {-Físicamente fuerte, Edad del em- } \\
\text { pleado, Libre de enfermedades }\end{array}$ \\
\hline & & Cumplimiento: & $\begin{array}{l}\text {-Cargos y litigios, cuestiones de } \\
\text { seguridad, quejas }\end{array}$ \\
\hline
\end{tabular}

Tabla 1. Capital Humano en Organizaciones/Empresas

Fuente: Elaboración propia.

De los indicadores presentados se rescata entre los más representativos a nivel empresarial componentes para su medición tales como la educación, salud, experiencia, capacitación, capacidades, habilidades, conocimientos, actitudes y saberes en general de los individuos. 


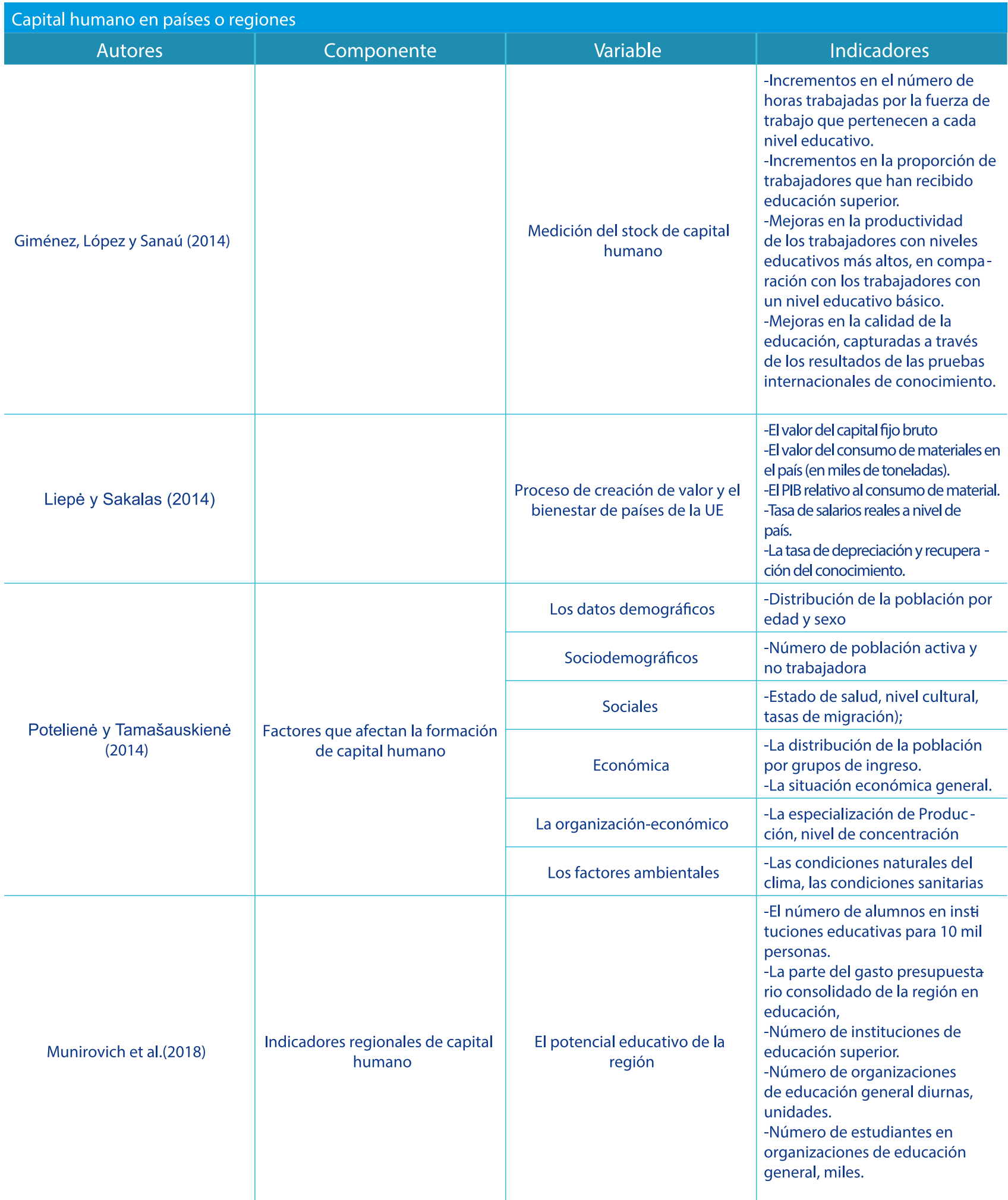


-La proporción de los gastos del presupuesto consolidado de la región en salud a GRP.

Potencial sanitario regional

- La esperanza de vida al nacer. -Morbilidad para 10.000 per sonas.

-Instalaciones hospitalarias, piezas. -Población

-Salario promedio -Nivel de actividad económica de la población.

Potencial laboral de la región. - La tasa de desempleo de la población.

-Población

-Producto regional bruto

-El número de espectadores de los teatros y el número de visitas al museo por cada 1000 perso nas. La población.

-El número de delitos registrados

Potencial sociocultural de la región. por cada 100 mil personas. la población.

-Venta de bebidas alcohólicas en términos físicos per cápita.

-Número de teatros profesionales, unidades.

-Número de instituciones de tipo cultural-ocio, unidades.

-La participación de las industrias de alta tecnología e intensivas en conocimiento en GRP, $\%$. -Proporción del gasto interno en investigación y desarrollo en Potencial intelectual de la región GRP,\%

-Número de patentes por cada 10 mil personas.

-El número de investigadores titulados en ciencias.

-El número de personal dedicado a la investigación y desarrollo.

-Porcentaje de empleados en actividades de alta tecnología y de tecnología media en el número total de empleados en la economía de la región,\%

Potencial de innovación de la región.

-Número de tecnologías de fabricación avanzadas creadas.

-La participación de bienes innovadores, obras, servicios en el volumen total de mercancías enviadas, trabajos realizados, servicios.

-Número de empleados en el ámbito de la actividad empresarial individual, mil personas.

-Participación de las ganancias de la propiedad intelectual, al total de GRP, \%

Potencial emprendedor

-Número de pequeñas empresas en la región.

-Programas regionales de apoyo al emprendimiento en la región, pcs.

-Participación de los ingresos por pequeñas empresas al total de GRP,\% 


\begin{tabular}{|c|c|c|c|}
\hline Autores & Componente & Variable & Indicadores \\
\hline $\begin{array}{l}\text { Olayiwola, Oseni y Segun } \\
\qquad(2018)\end{array}$ & & Desarrollo del capital humano & $\begin{array}{l}\text {-Inversión en el sector educativo. } \\
\text {-inversión en áreas de capacita - } \\
\text { ción de estudiantes en todos los } \\
\text { niveles. } \\
\text {-Número de capacitaciones, } \\
\text { programas y cursos profesiona- } \\
\text { les para el fomento del espíritu } \\
\text { empresarial. } \\
\text {-inversión en el sector salud. } \\
\text {-número de instalaciones de salud } \\
\text { con calidad en el país. } \\
\text {-niveles de desempleo } \\
\text {-niveles de pobreza en el país }\end{array}$ \\
\hline
\end{tabular}

Tabla 2. Capital humano en países o regiones

Fuente: Elaboración propia a partir de la revisión de literatura

En cuanto a los indicadores de capital humano a nivel país o región, se derivan para su medición algunos componentes más representativos tales como datos demográficos de la población, inversión en la educación y salud, instituciones de educación y salud, producto interno bruto, tasa de salarios, oportunidades laborales etc.

De manera más específica, para medir el desarrollo del capital humano en un país o región, el indicador que principalmente se ha considerado es el PIB, por otro lado los indicadores de Educación y salud, se pueden ver representados en ambas vertientes analizadas, de hecho la educación en efecto, ha sido considerada en la actualidad como un factor clave y de suma importancia para el desarrollo en todos los países del mundo, sin ir tan lejos, el nivel de educación (formación y/o experiencia) es el primer indicador que se toma en cuenta para medir el desarrollo del CH en una organización.

No obstante, de acuerdo a los indicadores propuestos por diversos autores en los artículos de investigación revisados, se puede apreciar que la mayoría de estos se asemejan o coinciden, y que varía en mayor medida el nivel de impacto (organización a país) para determinar el grado de desarrollo de su CH.

De esta manera es posible afirmar que los estudios para determinar el desarrollo de CH en las empresas, regiones o países han venido tomando como referencia este tipo de datos de índole cuantitativo como cualitativo, pues se han dado cuenta de que la vía más más probable para obtener mayores ventajas competitivas es mediante la inversión de su CH, mejorando en cuanto a eficiencia, productividad y competitividad. 


\section{CONCLUSIONES}

Como se logró identificar una vez iniciado el análisis de revisión de literatura, se identificó que existían comúnmente dos vertientes estudiadas en torno a los indicadores de $\mathrm{CH}$, por lo que se llegó a concluir en dos principales aportes, en primer lugar es interesante retomar que los pioneros clave en la historia del capital humano en sus inicios, han sido economistas, los cuales comenzaron a tomarle una gran importancia a información y conocimientos del recurso humano, pues se llegó a visualizar que estos son la clave para que las organizaciones adquieran y creen su propio conocimiento, viéndose reflejada después esta inversión en su desarrollo, en beneficios económicos para las organizaciones. Incluso como plantea, Rubio (2016) el capital humano es un elemento clave que actúa como combustible para el motor de la innovación y de desarrollo y por lo tanto para el crecimiento tanto económico como de posición de todo tipo de organizaciones.

Así mismo, en segundo lugar retomando uno de los aspectos centrales de este artículo, se logró también concluir en la identificación de dos vertientes, es decir indicadores de medición sobre el CH en organización/empresa y país/ región. Siendo importante además, mencionar que en ambas es el $\mathrm{CH}$ mediante el desarrollo de todo ese conjunto de elementos que conforman (formación, experiencia, habilidades, competencias, conocimientos, carácter innovador y propositivo) el que mueve cualquier organización y en conjunto al país entero. Lo cual más adelante podría impactar directamente en el éxito o fracaso de cualquier organismo, deduciendo entonces al $\mathrm{CH}$ como la mejor inversión, tanto para cualquier tipo de organización como para países en su conjunto.

Por último, se recomienda retomar el conjunto de los indicadores descritos en este trabajo como base para la elaboración de una propuesta de instrumento de medición para el estudio de nuevas investigaciones, sin embargo, es importante dejar en claro la existencia de las dos vertientes aportadas en esta investigación, debido a la confusión que se pudiera generar al momento de realizar un estudio sobre el tema. 


\section{REFERENCIAS}

Abrigo, M., Lee, S., \& Park, D. (2017). Human Capital Spending, Inequality, and Growth in Middle-Income Asia. ADB Economics Working Paper Series, (529), 1-20. DOI: http://dx.doi.org/10.22617/WPS179147-2

Aronson, P. (2007). El retorno de la teoría del capital humano. Universidad nacional de San Luis-Argentina, VIII(2), 9-26. Recuperado de https://dialnet.unirioja.es/descarga/articulo/2693624.pdf

Bermúdez, M., Boscan, N., Muñoz, D., Vidal, B., \& Archila, C. (2017). Gestión del conocimiento en grupos de I+D: un enfoque basado en los componentes del capital humano. Revista lasallista de investigación, 14(1), 133-143. DOI: $10.22507 /$ rli.v $14 n 1$ a 12

Bueno, E., Del Real, H., Fernández, P., Longo, M., Merino, C., Murcia, C., \& y Salmador, M. (2011). Modelo Intellectus de medición, gestión e información del capital intelectual. Recuperado de https://www.researchgate.net/ publication/298346530_Modelo_Intellectus_Medicion_y_Gestion_del_Capital_Intelectual

Cardona, M., Montes, I., Vásquez, I., Villegas, M., \& Brito, T. (2007). Capital humano: una mirada desde la educación y la experiencia laboral. Medellín: Universidad EAFIT. Recuperado de http://publicaciones.eafit.edu.co/index.php/ cuadernos-investigacion/article/download/1287/1166

Chadwick, C. (2017). Toward a more comprehensive model of firms' human capital rents. Academy of Management Review, 42(3), 499-519. https://doi.org/10.5465/amr.2013.0385

Demuner, M., Saavedra, M., \& Camarena, M. (2017). Medición del capital intelectual en el sector bancario: aplicación de los modelos Skandia y VAIC. Innovar. Revista de Ciencias Administrativas y Sociales, 27 (66), 75-89. http://dx.doi. org/10.15446/innovar.v27n66.66712

Fink, A. (2014). Conducting research literature reviews from the internet to paper. Los Angeles: Sage.

Ganga, F., Cassinelli, A., Díaz, G., \& Maluk, S. (2016). Breves disquisiciones teóricas en torno al tema capital humano y eficiencia organizativa. Revista Gaceta Laboral, 22(1), 27-39. Recuperado de http://www.redalyc.org/articulo. oa?id=33646908002

García, M, \& Chávez, R. (2017). Indicadores para medir el capital humano en micro, pequeñas y medianas empresas en México. Revista CIMEXUS, XII(2) 2017. 167-178. Recuperado de https://dialnet.unirioja.es/descarga/ articulo/6317395.pdf

Giménez, G., López-Pueyo, C., \& Sanaú, J. (2014). Medición del capital humano en los países OCDE y su relación con el crecimiento del PIB y la innovación. Revista de Economía Mundial, (39), 77-108.

Greer, C., Lusch, R., \& Hitt, M. (2017). A service perspective for human capital resources: a critical base for strategy implementation. Academy of Management Perspectives, 31(2), 137-158. https://doi.org/10.5465/amp.2016.0004

Herrera, S. (2010). La importancia de la educación en el desarrollo: la teoría del capital humano y el perfil edad ingresos por nivel educativo en viedma y carmen de patagones, argentina. Revista Pilquen, XII(13), 1-9. Recuperado de www.scielo.org.ar/pdf/spilquen/n13/n13a07.pdf

Heuermann, D. (2011). Human Capital Externalities in Western Germany. Spatial Economic Analysis, 6(2), $139-165$. DOI: $10.1080 / 17421772.2011 .557775$

Israelsen, R., \& Yonker, S. (2017). Key Human Capital. Journal of financial and quantitative analysis, 52(1), $175-214$. doi: $10.1017 /$ s0022109016000880

Iwamoto, H., \& Takahashi, M. (2015). A Quantitative Approach to Human Capital Management. Procedia, Social and Behavioral Sciences, 172, 112-119. doi: 10.1016/j.sbspro.2015.01.343 
Liep̌é, Z., \& Sakalas, A. (2014). Evaluation of human capital role in the value creation process. Procedia, Social and Behavioral Sciences 156, 78-82. DOI: 10.1016 / j.sbspro.2014.11.123

Lizote, S., Ribeiro, C., Verdinelli, M., \& Terres, J. (2017). Human capital and its relationship with the organizational performance in accounting services providers. Revista Catarinense da Ciência Contábil, 16(48), 23-26. doi: $10.16930 / 2237-7662 /$ rccc.v16n48.2373

López, A., Ojeda, J., \& Ríos, M. (2017). La responsabilidad social empresarial desde la percepción del capital humano. Estudio de un caso. Revista de Contabilidad - Spanish Accounting Review, 20(1)36-46. http://dx.doi.org/10.1016/j. rcsar.2016.01.001

Mačiulytè, A., \& Matuzeviciutè, K. (2018). Impact of human capital development on productivity growth in UE member states. Business, Management and Education, 16(1), 1-12. https://doi.org/10.3846/bme.2018.66

Mubarik, M., Chandran, V., \& Devadason, E. (2017). Measuring Human Capital in Small and Medium Manufacturing Enterprises: What Matters? Springer, 137 (2), 605-623. DOI 10.1007/s1 1205-017-1601-9

Münch, L. (2017) Administración de capital humano: la gestión del activo más valioso de la organización. México: Trillas.

Munirovich, E., Ilfarovich, R., Olegovych, V., Sergeevna, I., Sultangalievich, A., Andreevich, E., \& Mikhailovna, N. (2018). Research of human capital and its potential management on the example of regions of the russian federation. Journal of Entrepreneurship Education, 21(2), 1-14.

Nnebedum, C., \& Akinfolarin, V. (2017). Principals' Human Capital Development Practices For Enhancing Staff Personnel Administration In Secondary Schools In Oyo State, Nigeria. International Journal of Advanced Research and Publications, 1(5), 356-351. Recuperado de https://eric.ed.gov/?id=ED580925

Olayiwola, A., Oseni, I., \& Segun, A. (2018). Human Capital Development: A Catalyst for Achieving Sdgs in Nigeria. CECONOMICA, 14(4), 25-41.

Pérez-Fuentes, D., \& Castillo-Loaiza, J. (2016). Capital humano, teorías y métodos: importancia de la variable salud. Economía, sociedad y territorio, 52, 651-673. www.scielo.org.mx/pdf/est/v16n52/2448-6183-est-16-52-00651.pdf

Potelienè, S., \& Tamašauskienè, Z (2014). Zmogiškojo kapitalo konceptualizacija: raida, samprata ir formavimas. Verslo sistemos ir ekonomika business systems and ECONOMICS, 4(1), 89-106. doi:10.13165/VSE-14-4-1-08

Raffiee, J., \& Coff, R. (2016). Micro-foundations of firm-specific human Capital: when do employees perceive their skills to be firm-specific? Academy of Management Journal, 59(3), 766-790. http://dx.doi.org/10.5465/amj.2014.0286

Rocha, F., Genari, D., \& Macke, J. (2018). A obsolescência do capital humano nas empresas de tecnologia da informação: um estudo na associação internetsul. Revista Alcance, 24(4), 513-534. Doi: alcance.v24n4(Out/Dez).p513-534

Rubio, A. (2016). Capital humano y sociedad de control. Cuadernos de Filosofía Latinoamericana, 37(115), $103-126$. DOI: http://dx.doi.org/10.15332/s0120-8462.2016.0115.04

Sweetland, S. R. (1996). Human Capital Theory: Foundations of a Field of Inquiry. Review of Educational Research, 66(3), 341-359. doi:10.3102/00346543066003341

Thindisa, L., \& Urban, B. (2018) Human-social capital and market access factors influencing agro-processing participation by small-scale agripreneurs: The moderating effects of transaction costs. Acta Commercii, 18(1), 1-10. https://doi.org/10.4102/ ac.v18i1.500

Villarreal, E. (2016). Externalidades del capital humano en México. El trimestre económico, 4(332), 747-788. http:// dx.doi.org/10.20430/ete.v83i332.238 